\title{
New Analytical and Numerical Solutions for Mixed Convection Boundary-Layer Nanofluid Flow along an Inclined Plate Embedded in a Porous Medium
}

\author{
Emad H. Aly ${ }^{1,2}$ and Abdelhalim Ebaid ${ }^{3}$ \\ ${ }^{1}$ Department of Mathematics, Faculty of Science, King Abdulaziz University, Jeddah 21589, Saudi Arabia \\ ${ }^{2}$ Department of Mathematics, Faculty of Education, Ain Shams University, Roxy, Cairo 11757, Egypt \\ ${ }^{3}$ Department of Mathematics, Faculty of Science, University of Tabuk, Tabuk 71491, Saudi Arabia
}

Correspondence should be addressed to Emad H. Aly; emad-aly@hotmail.com

Received 17 July 2013; Accepted 23 August 2013

Academic Editor: Mohamed Fathy El-Amin

Copyright (C) 2013 E. H. Aly and A. Ebaid. This is an open access article distributed under the Creative Commons Attribution License, which permits unrestricted use, distribution, and reproduction in any medium, provided the original work is properly cited.

\begin{abstract}
Two different analytical and numerical methods have been applied to solve the system describing the mixed convection boundarylayer nanofluids flow along an inclined plate embedded in a porous medium, namely, homotopy perturbation method (HPM) and Chebyshev pseudospectral differentiation matrix (ChPDM), respectively. Further, ChPDM is used as a control method to check the accuracy of the results obtained by HPM. The analytical method is applied using a new way for the deformed equations, and the resulted solution was expressed in terms of a well-known entire error function. In addition, using only two terms of the homotopy series, the approximate analytical solution is compared with the numerical solution obtained by the accurate ChPDM approach. The results reveal that good agreements have been achieved between the two approaches for various values of the investigated physical parameters.
\end{abstract}

\section{Introduction}

The concept of nanofluids is put into practice particularly after the tremendous development of nanotechnologies in the last decade, as they are of great importance in many industrial and engineering applications, especially in heat transfer enhancement [1-4]. Nanofluids are produced by dispersing nanometer-scale solid particles into base liquids such as water, ethylene glycol, and oils. Normally, if the particle sizes are in the $1-100 \mathrm{~nm}$ ranges, they are generally called nanoparticles.

Recently, the boundary-layer flow of nanofluids has attracted much attention [5-8]. The flow field of such problems is governed at first by a system of linear and nonlinear partial differential equations which are then transformed into a new system of linear and non-linear ordinary differential equations with applying the appropriate transformations. Due the difficulties that arise in obtaining the analytical solutions for the resulted system, most of the authors resort to numerical methods.

Although the numerical methods have the upper hand over the analytical methods in solving the complex systems of differential equations, the analytical solutions can be viewed as an optimal choice when available. In addition, the obtained solutions can be directly used for getting the required plots without needing subprograms as in the numerical methods. In conclusion, one can say that each of the two approaches has its own advantage over the other. Therefore, comparing the results obtained by two different analytical and numerical approaches is generally of great importance in the applied sciences. Two different analytical methods (homotopy perturbation method, HPM) [9-18] and numerical method (Chebyshev pseudospectral differentiation matrix, ChPDM) [19-21] are proposed in this paper for the mixed convection boundary-layer nanofluids flow along an inclined plate embedded in a porous medium. 
More details about these techniques and their advantages are introduced in the next two paragraphs.

The homotopy perturbation method (HPM) was proposed by $\mathrm{He}$ [9-15] as an analytical technique to solve nonlinear differential equations. This method has been widely used by many authors to investigate various models [16-18]. Unlike, the requirement for the regular perturbation techniques [19], the homotopy perturbation method is always valid no matter whether there exists small physical parameter or not. It combines the traditional perturbation method and the homotopy technique to deform a nonlinear problem into a simple solving one. The solution using this method is expressed as the summation of an infinite series, which usually converges rapidly to the exact solution in most cases. Several terms of the series solution can be used for approximating the exact solution with a high degree of accuracy.

On the other hand, the ChPDM is a known highly accurate numerical approach which was introduced and successfully applied by Aly et al. [20] to analyze the twodimensional MHD boundary-layer flow over a permeable surface with a power law stretching velocity in the presence of a magnetic field applied normally to the surface. Under certain circumstances, it is shown that the problem has an infinite number of solutions which were examined by this technique. Further, Guedda et al. [21] have applied this method to validate and evidence the analysis of twodimensional mixed convection boundary-layer flow over a vertical flat plate embedded in a porous medium saturated with water at $4^{\circ} \mathrm{C}$ (maximum density) and applied magnetic field. Both cases of the assisting and opposing flows are considered. Multiple similarity solutions are obtained and investigated by ChPDM under the power law variable wall temperature, or variable heat flux, or variable heat transfer coefficient. Recently, Aly and Ebaid [22] have applied this technique to solve the current problem in the absence of the magnetic and permeability of parameters. They found that the ChPDM results are very accurate in an excellent manner compared to those published in the literature using the homotopy analysis [23]. It is then successfully applied to validate and evidence the resulted exact solutions for different positive and negative values of the investigated parameters.

The motivation of presenting this paper is therefore to extend the applicability of both HPM and ChPDM approaches. The suggested procedure is based first on obtaining all the possible exact solutions. Then, the second step of the suggested procedure is to validate these results numerically to explore the effectiveness and efficiency of the proposed numerical approach. Besides, comparisons with other published results are also presented, where a full agreement is observed. In addition, it is shown that the solution up to the first order is sufficient to obtain accurate results.

\section{The Physical Model}

Rana et al. [24] considered the steady, incompressible, laminar, boundary-layer flow of a nanofluid along a semi-infinite inclined flat plate in a nanofluid-saturated porous medium with an acute angle $\alpha$ to the vertical. The mathematical model is governed by the following system of nonlinear ordinary differential equations:

$$
\begin{gathered}
f^{\prime \prime}=\frac{\mathrm{Ra}_{x}}{\mathrm{Pe}_{x}}\left(\theta^{\prime}-N_{r} \phi^{\prime}\right) \cos (\alpha), \\
\theta^{\prime \prime}+\frac{1}{2} f \theta^{\prime}+N_{b} \phi^{\prime} \theta^{\prime}+N_{t}\left(\theta^{\prime}\right)^{2}=0, \\
\phi^{\prime \prime}+\frac{1}{2} \operatorname{Le} f \phi^{\prime}+\frac{N_{t}}{N_{b}} \theta^{\prime \prime}=0,
\end{gathered}
$$

which has to be solved, subject to the boundary conditions:

$$
\begin{array}{ll}
f(0)=0, & f^{\prime}(\infty)=1, \\
\theta(0)=1, & \theta(\infty)=0, \\
\phi(0)=1, & \phi(\infty)=0,
\end{array}
$$

where primes denoting differentiation with respect to $\eta$ and Le, $N_{r}, N_{b}, N_{t}, \mathrm{Ra}_{x}$, and $\mathrm{Pe}_{x}$ are the Lewis number, buoyancy ratio parameter, Brownian motion parameter, thermophoresis parameter, local Darcy-Rayleigh number, and local Péclet number, respectively. On integrating (1) with respect to $\eta$ from $\infty$ to $\eta$ and using the boundary conditions in (3)-(4), we have

$$
f^{\prime}=1+\Omega\left(\theta-N_{r} \phi\right), \quad \text { where } \Omega=\frac{\mathrm{Ra}_{x}}{\mathrm{Pe}_{x}} \cos (\alpha) .
$$

\section{Application of the HPM}

In this section, the homotopy perturbation method (HPM) is applied to search for the approximate analytical solutions for the system of (6) and (2) with the boundary conditions (3)(5). In order to apply this method, we rewrite the investigated system in terms of an embedding parameter $p$ as

$$
\begin{gathered}
f^{\prime}=1+p\left[\Omega\left(\theta-N_{r} \phi\right)\right], \\
\theta^{\prime \prime}+\frac{1}{2} f \theta^{\prime}=-p\left[N_{b} \phi^{\prime} \theta^{\prime}+N_{t}\left(\theta^{\prime}\right)^{2}\right], \\
\phi^{\prime \prime}+\frac{1}{2} \operatorname{Le} f \phi^{\prime}=-p\left[\frac{N_{t}}{N_{b}} \theta^{\prime \prime}\right] .
\end{gathered}
$$

According to the HPM, the following assumptions are assumed:

$$
\begin{aligned}
& f(\eta)=\sum_{n=0}^{\infty} p^{n} f_{n}(\eta), \\
& \theta(\eta)=\sum_{n=0}^{\infty} p^{n} \theta_{n}(\eta), \\
& \phi(\eta)=\sum_{n=0}^{\infty} p^{n} \phi_{n}(\eta) .
\end{aligned}
$$


Substituting (8) into (7) and by comparing the like powers of the embedding parameter $p^{0}$, we get the following systems:

$$
\begin{array}{rlrl}
f_{0}^{\prime}(\eta)=1, & f_{0}(0)=0, \\
\theta_{0}^{\prime \prime}+\frac{1}{2} f_{0} \theta_{0}^{\prime}=0, & \theta_{0}(0)=1, & \theta_{0}(\infty)=0, \\
\phi_{0}^{\prime \prime}+\frac{1}{2} f_{0} \phi_{0}^{\prime}=0, & \phi_{0}(0)=1, & \phi_{0}(\infty)=0,
\end{array}
$$

$$
\phi_{n+1}^{\prime \prime}+\frac{1}{2} \operatorname{Le} f_{0} \phi_{n+1}^{\prime}=-\frac{N_{t}}{N_{b}} \theta_{n}^{\prime \prime}-\frac{1}{2} \operatorname{Le} \sum_{k=0}^{n} f_{n+1-k} \phi_{k}^{\prime} .
$$

The last systems are subjected to the boundary conditions:

$$
\begin{gathered}
f_{n+1}(0)=0, \\
\theta_{n+1}(0)=0, \quad \theta_{n+1}(\infty)=0, \\
\phi_{n+1}(0)=0, \quad \phi_{n+1}(\infty)=0 .
\end{gathered}
$$

3.1. Solution of the Zeroth-Order System. The exact solutions of the zeroth-order system are given as

$$
\begin{gathered}
f_{0}(\eta)=\eta, \\
\theta_{0}(\eta)=1-\operatorname{erf}\left(\frac{1}{2} \eta\right), \\
\phi_{0}(\eta)=1-\operatorname{erf}\left(\sqrt{\frac{\mathrm{Le}}{4} \eta}\right),
\end{gathered}
$$

where erf is the error function; see Abramowitz and Stegun [25]. These exact solutions can be verified by the direct substitution.

3.2. Solution of the First-Order System. The system of firstorder is obtained by considering $n=0$ in (10) and given by

$$
\begin{gathered}
f_{1}^{\prime}(\eta)=\theta_{0}-N_{r} \phi_{0}, \\
\theta_{1}^{\prime \prime}+\frac{1}{2} f_{0} \theta_{1}^{\prime}=-\frac{1}{2} f_{1} \theta_{0}^{\prime}-\left[N_{b} \phi_{0}^{\prime} \theta_{0}^{\prime}+N_{t}\left(\theta_{0}^{\prime}\right)^{2}\right], \\
\phi_{1}^{\prime \prime}+\frac{1}{2} \operatorname{Le} f_{0} \phi_{1}^{\prime}=-\frac{1}{2} \operatorname{Le} f_{1} \phi_{0}^{\prime}-\frac{N_{t}}{N_{b}} \theta_{0}^{\prime \prime},
\end{gathered}
$$

where the appropriated boundary conditions are

$$
\begin{gathered}
f_{1}(0)=0, \\
\theta_{1}(0)=0, \quad \theta_{1}(\infty)=0, \\
\phi_{1}(0)=0, \quad \phi_{1}(\infty)=0 .
\end{gathered}
$$

On using the initial condition (14), the exact solution of the $f_{1}$-equation in system (13) is given as follows:

$$
\begin{aligned}
& f_{1}(\eta)=\Omega \int_{0}^{\eta}\left[\theta_{0}(\sigma)-N_{r} \phi_{0}(\sigma)\right] d \sigma \\
& =\left(1-N_{r}\right) \Omega \eta \\
& +\Omega \int_{0}^{\eta}\left[N_{r} \operatorname{erf}\left(\sqrt{\frac{\mathrm{Le}}{4}} \sigma\right)-\operatorname{erf}\left(\frac{1}{2} \sigma\right)\right] d \sigma \\
& =\sqrt{\frac{4}{\pi}}\left(1-\frac{N_{r}}{\sqrt{\mathrm{Le}}}\right) \Omega \\
& +\Omega\left[\left(\theta_{0}-N_{r} \phi_{0}\right) \eta+\sqrt{\frac{4}{\pi}}\right. \\
& \left.\times\left(\frac{N_{r}}{\sqrt{\mathrm{Le}}} e^{-(1 / 4) \mathrm{Le}^{2}}-e^{-(1 / 4) \eta^{2}}\right)\right] .
\end{aligned}
$$

Regarding the view of $\theta_{1}$-equation in (13) and boundary conditions (15), we obtain $\theta_{1}$ in the following closed form:

$$
\theta_{1}(\eta)=I_{1}(\eta)-I_{1}(\infty) \operatorname{erf}\left(\frac{1}{2} \eta\right)
$$

where $I_{1}(\eta)$ is given by

$$
I_{1}(\eta)=\int_{0}^{\eta} e^{-(1 / 4) \sigma^{2}} d \sigma \int_{0}^{\sigma} e^{(1 / 4) \xi^{2}} \psi_{1}(\xi) d \xi,
$$

where

$$
\psi_{1}(\xi)=-\left[\frac{1}{2} f_{1}(\xi)+N_{b} \phi_{0}^{\prime}(\xi) \theta_{0}^{\prime}(\xi)+N_{t}\left(\theta_{0}^{\prime}(\xi)\right)\right] .
$$

The double integral in (19) can be expressed in terms of single integrations by using the integral identity:

$$
\int_{0}^{\eta} g(\sigma) d \sigma \int_{0}^{\sigma} h(\xi) d \xi=\int_{0}^{\eta} h(\xi) d \xi \int_{\xi}^{\eta} g(\sigma) d \sigma,
$$

and hence,

$$
\begin{aligned}
I_{1}(\eta)= & \int_{0}^{\eta} e^{(1 / 4) \xi^{2}} \psi_{1}(\xi) d \xi \int_{\xi}^{\eta} e^{-(1 / 4) \sigma^{2}} d \sigma \\
= & \sqrt{\pi} \int_{0}^{\eta}\left[\operatorname{erf}\left(\frac{1}{2} \eta\right)-\operatorname{erf}\left(\frac{1}{2} \xi\right)\right] e^{(1 / 4) \xi^{2}} \psi_{1}(\xi) d \xi \\
= & \sqrt{\pi} \operatorname{erf}\left(\frac{1}{2} \eta\right) \int_{0}^{\eta} e^{(1 / 4) \xi^{2}} \psi_{1}(\xi) d \xi \\
& -\sqrt{\pi} \int_{0}^{\eta} e^{(1 / 4) \xi^{2}} \operatorname{erf}\left(\frac{1}{2} \xi\right) \psi_{1}(\xi) d \xi .
\end{aligned}
$$

In a similar way, we obtain the solution of the $\phi_{1}$-equation in (13) with the boundary conditions (16) in closed form as follows:

$$
\phi_{1}(\eta)=I_{2}(\eta)-I_{2}(\infty) \operatorname{erf}\left(\sqrt{\frac{\mathrm{Le}}{4}} \eta\right)
$$


where $I_{2}(\eta)$ is given by

$$
I_{2}(\eta)=\int_{0}^{\eta} e^{-(1 / 4) \operatorname{Le} \sigma^{2}} d \sigma \int_{0}^{\sigma} e^{(1 / 4) \operatorname{Le} \xi^{2}} \psi_{2}(\xi) d \xi
$$

where

$$
\psi_{2}(\xi)=-\left[\frac{1}{2} \operatorname{Le} f_{1}(\xi) \phi_{0}^{\prime}(\xi)+\frac{N_{t}}{N_{b}} \theta_{0}^{\prime \prime}(\xi)\right] .
$$

Following the above analysis of $I_{1}(\eta)$ in $(22), I_{2}(\eta)$ can be given as

$$
\begin{aligned}
I_{2}(\eta)= & \sqrt{\frac{\pi}{\mathrm{Le}}} \operatorname{erf}\left(\sqrt{\frac{\mathrm{Le}}{4} \eta}\right) \int_{0}^{\eta} e^{(1 / 4) \mathrm{Le} \xi^{2}} \psi_{2}(\xi) d \xi \\
& -\sqrt{\frac{\pi}{\mathrm{Le}}} \int_{0}^{\eta} e^{(1 / 4) \mathrm{Le} \xi^{2}} \operatorname{erf}\left(\sqrt{\frac{\mathrm{Le}}{4}} \xi\right) \psi_{2}(\xi) d \xi .
\end{aligned}
$$

Therefore, at $p=1$, the approximate solutions for the temperature distribution $\theta(\eta)$ and the nanoparticle concentration $\phi(\eta)$ up to the first order are given by

$$
\begin{gathered}
\theta(\eta) \approx 1-\operatorname{erf}\left(\frac{1}{2} \eta\right)+I_{1}(\eta)-I_{1}(\infty) \operatorname{erf}\left(\frac{1}{2} \eta\right), \\
\phi(\eta) \approx 1-\operatorname{erf}\left(\sqrt{\frac{\mathrm{Le}}{4} \eta}\right)+I_{2}(\eta)-I_{2}(\infty) \operatorname{erf}\left(\sqrt{\frac{\mathrm{Le}}{4} \eta}\right) .
\end{gathered}
$$

The current approximate solution obtained in this section is checked via comparing with those obtained in the literature.

3.3. Special Case: Horizontal Plate. At $\alpha=\pi / 2$, we have the following simple expressions for the temperature distribution $\theta(\eta)$ and the nanoparticle concentration distribution $\phi(\eta)$ :

$$
\begin{aligned}
\theta(\eta)= & 1-\operatorname{erf}\left(\frac{1}{2} \eta\right)+\left[N_{b} I_{3}(\infty)+N_{t}\right] \operatorname{erf}\left(\frac{1}{2} \eta\right) \\
& -N_{b} I_{3}(\eta)-N_{t}\left[\operatorname{erf}\left(\frac{1}{2} \eta\right)\right]^{2}, \\
\phi(\eta)= & 1-\operatorname{erf}\left(\frac{\sqrt{\mathrm{Le}}}{2} \eta\right)+\frac{2 N_{t}}{N_{b}(\mathrm{Le}-1)} \\
& \times\left[\operatorname{erf}\left(\frac{\sqrt{\mathrm{Le}}}{2} \eta\right)-\operatorname{erf}\left(\frac{1}{2} \eta\right)\right],
\end{aligned}
$$

where

$$
I_{3}(\eta)=\frac{2}{\sqrt{\pi}} \int_{0}^{\eta} e^{-\sigma^{2} / 4} \operatorname{erf}\left(\frac{\sqrt{\mathrm{Le}}}{2} \sigma\right) d \sigma .
$$

\section{The ChPDM Technique}

Chebyshev pseudospectral differentiation matrix (ChPDM) technique is briefly introduced; see Guedda et al. [21], Aly and Ebaid [22], and Van Gorder et al. [23]. On supposing that the domain of the problem is $\left[0, \eta_{\infty}\right]$, then the following algebraic mapping $z=2 \eta / \eta_{\infty}-1$ transfers the domain to the Chebyshev one, that is $[-1,1]$. It is known that the Chebyshev polynomials are usually taken with their associated collocation points in the interval $[-1,1]$ given by

$$
z_{j}=\cos \left(\frac{\pi}{N} j\right), \quad j=0,1, \ldots, N
$$

Therefore, the $k$ th derivative of any function, say $\mathbf{F}(z)$, at these collocation points can be approximated by the equation:

$$
\mathbf{F}^{(k)}=D^{(k)} \mathbf{F}
$$

where $D^{(k)} \mathbf{F}$ is the Chebyshev pseudospectral approximation of $\mathbf{F}^{(k)}$, where $\mathbf{F}=\left[F\left(z_{0}\right), F\left(z_{1}\right), \ldots, F\left(z_{N}\right)\right]^{T}$ and $\mathbf{F}^{(k)}=$ $\left[F^{(k)}\left(z_{0}\right), F^{(k)}\left(z_{1}\right), \ldots, F^{(k)}\left(z_{N}\right)\right]^{T}$. The entries of the matrix $D^{(k)}$ are given by

$$
\begin{aligned}
d_{i, j}^{(k)}=\frac{2 \varphi_{j}}{N} \sum_{r=k}^{N} \sum_{\substack{n=0 \\
(n+r-k) \text { even }}}^{r-k} \phi_{r} b_{n, r}^{k}(-1)^{[(r j+n i) / N]} \\
\\
\quad \times z_{r j-N[r j / N]} z_{n i-N[n i / N]}
\end{aligned}
$$

where $\varphi_{j}=1$, except for $\varphi_{0}=\varphi_{N}=1 / 2$, and

$$
b_{n, r}^{k}=\frac{2^{k} r}{(k-1) ! c_{n}} \frac{(v-n+k-1) !(v+k-1) !}{(v) !(v-n) !}
$$

where $2 v=r+n-k$ and $c_{0}=2, c_{j}=1, j \geq 1$. The elements $d_{0,1}^{(k)}$ are the major elements concerning its values. Accordingly, they bear the major error responsibility when compared to the other elements. It is shown that the error in $d_{0,1}^{(1)}$ is of order $O\left(N^{2} \varepsilon_{r}\right)$, where $\varepsilon_{r}$ is the machine precision [26].

On the view of [21-23], derivatives of the function $f(\eta)$, $\theta(\eta)$, and $\phi(\eta)$ at the points $z_{i}$ are given by

$$
\begin{array}{r}
\chi^{(k)}\left(z_{i}\right)=\sum_{j=0}^{N} d_{i, j}^{(k)} \chi\left(z_{j}\right), \quad \chi \equiv f, \theta, \phi, k=1,2,3, \\
i=1,2, \ldots, N .
\end{array}
$$


Therefore, (1)-(5) become, respectively,

$$
\begin{gathered}
\sum_{j=0}^{N} d_{i, j}^{(2)} f\left(z_{j}\right)=\frac{\mathrm{Ra}_{x}}{\mathrm{Pe}_{x}}\left[\sum_{j=0}^{N} d_{i, j}^{(1)} \theta\left(z_{j}\right)-N_{r} \sum_{j=0}^{N} d_{i, j}^{(1)} \phi\left(z_{j}\right)\right] \cos \alpha, \\
\sum_{j=0}^{N} d_{i, j}^{(2)} \theta\left(z_{j}\right)+\frac{1}{2} f\left(z_{i}\right)\left(\frac{\eta_{\infty}}{2}\right) \sum_{j=0}^{N} d_{i, j}^{(1)} \theta\left(z_{j}\right)+N_{b} \sum_{j=0}^{N} d_{i, j}^{(1)} \theta\left(z_{j}\right) \\
\times \sum_{j=0}^{N} d_{i, j}^{(1)} \phi\left(z_{j}\right)+N_{t}\left(\sum_{j=0}^{N} d_{i, j}^{(1)} \theta\left(z_{j}\right)\right)^{2}=0, \\
\sum_{j=0}^{N} d_{i, j}^{(2)} \phi\left(z_{j}\right)+\frac{1}{2} \operatorname{Le} f\left(z_{i}\right)\left(\frac{\eta_{\infty}}{2}\right) \sum_{j=0}^{N} d_{i, j}^{(1)} \phi\left(z_{j}\right) \\
+\frac{N_{t}}{N_{b}} \sum_{j=0}^{N} d_{i, j}^{(2)} \theta\left(z_{j}\right)=0, \\
f\left(z_{N}\right)=0, \quad \sum_{j=0}^{N} d_{0, j}^{(1)} f\left(z_{j}\right)=0, \\
\theta\left(z_{N}\right)=1, \quad \theta\left(z_{0}\right)=0, \\
\phi\left(z_{N}\right)=1, \quad \phi\left(z_{0}\right)=0 .
\end{gathered}
$$

\section{Results and Discussion}

In Sections 3 and 4, mixed convection boundary-layer nanofluid flow along an inclined plate embedded in a porous medium has been solved analytically using two terms of the HPM and numerically by the ChPDM technique, respectively. The solutions were obtained for the temperature distribution $\theta$ and nano-particle concentration distribution $\phi$. In the current section, we aim to compare the results derived from HPM by those obtained by the ChPDM, which may be considered as a reference approach because it was successfully compared before with homotopy analysis method in [22]. In each forthcoming figure, it should be noted that solid and dotted curves refer to the results obtained by ChPDM and HPA, respectively.

Figure 1 shows the comparison between the HPM and ChPDM solutions for the nanoparticle concentration distribution $\phi(\eta)$ at different values of Brownian motion parameter $N_{b}$, where $N_{t}=0.5, N_{r}=0.5, \mathrm{Le}=10, \mathrm{Ra}_{x} / \mathrm{Pe}_{x}=0.5$, and $\alpha=\pi / 6$. From this figure, it is clear that the profiles of $\phi(\eta)$ are very close to each other and to those obtained in Figure 3 by Rana et al. [24].

The temperature distribution $\theta$ is depicted against $\eta$ at different values of thermophoresis parameter $N_{t}$ for $N_{b}=0.5$, $N_{r}=0.5, \mathrm{Le}=10, \mathrm{Ra}_{x} / \mathrm{Pe}_{x}=0.5$, and $\alpha=\pi / 6$ in Figure 2. It is also observed from it that excellent agreement is achieved on comparing with Figure 4 in [24]. These results may refer to that two terms approximate solutions of the HPM are sufficient to give accurate numerical results.

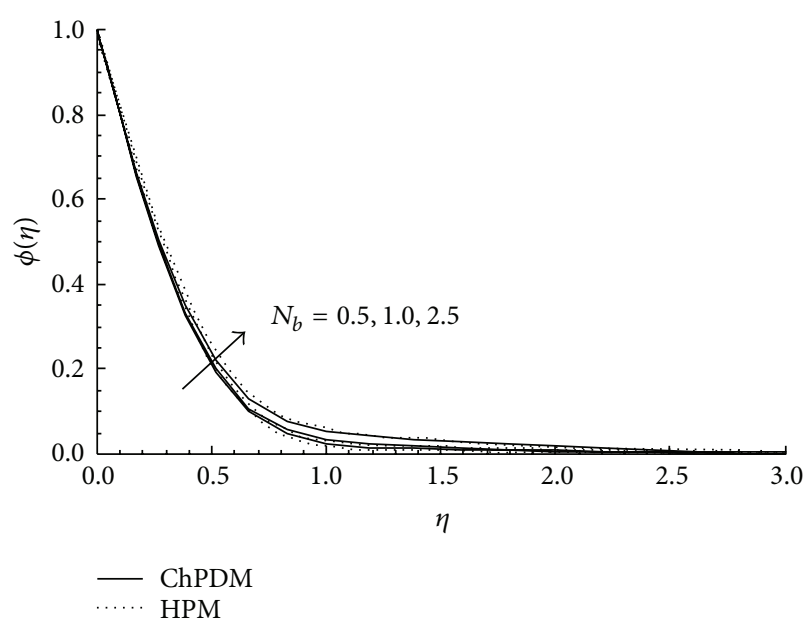

FIGURE 1: Comparison of ChPDM (solid) and HPM (dotted) for the effect of the Brownian motion parameter $\left(N_{b}\right)$ on the nanoparticle concentration distribution $\phi(\eta)$ at $N_{t}=0.5, N_{r}=0.5, \mathrm{Le}=10$, $\mathrm{Ra}_{x} / \mathrm{Pe}_{x}=0.5$, and $\alpha=\pi / 6$.

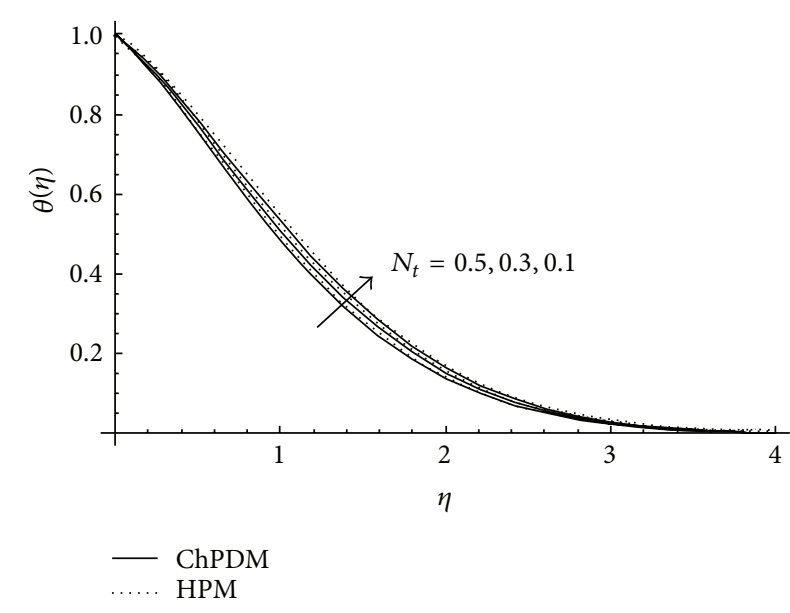

FIGURE 2: Comparison of ChPDM (solid) and HPM (dotted) for the effect of the thermophoretic parameter $\left(N_{t}\right)$ on the temperature distribution $\theta(\eta)$ at $N_{b}=0.5, N_{r}=0.5, \mathrm{Le}=10, \mathrm{Ra}_{x} / \mathrm{Pe}_{x}=0.5$, and $\alpha=\pi / 6$.

The last conclusion is also detected in Figure 3 for the effect of the buoyancy ratio parameter $N_{r}$ and the mixed convection parameter $\mathrm{Ra}_{x} / \mathrm{Pe}_{x}$ on the temperature distribution $\theta(\eta)$ at $N_{b}=0.5, N_{t}=0.5, \mathrm{Le}=10$, and $\alpha=\pi / 6$. From this figure, we can notice excellent agreements between the two approaches and further of those plotted in Figure 6 by Rana et al. [24].

Figure 4 indicates the effect of the inclination angle $\alpha$ on the nanoparticle concentration distribution $\phi$ at $N_{b}=0.5$, $N_{t}=0.5, N_{r}=0.5, \mathrm{Le}=10$, and $\mathrm{Ra}_{x} / \mathrm{Pe}_{x}=0.5$. It can be easily observed from this figure that the slight difference between the curves obtained via the two approaches increases with increasing $\alpha$, especially at $\alpha=\pi / 2$. A similar observation is detected from Figure 5 for the effect of Lewis number (Le) on $\phi$ at $N_{b}=0.5, N_{t}=0.5, N_{r}=0.5, \mathrm{Ra}_{x} / \mathrm{Pe}_{x}=0.5$, and 


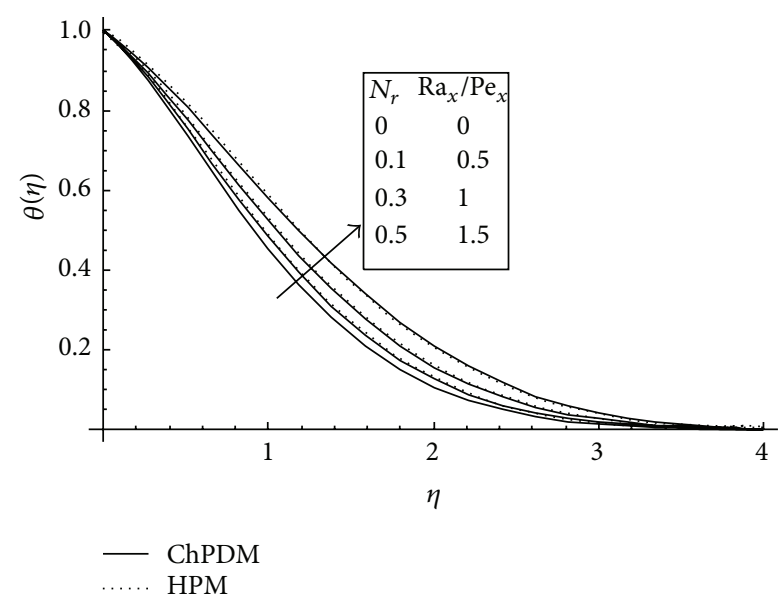

Figure 3: Comparison of ChPDM (solid) and HPM (dotted) for the effect of the buoyancy ratio parameter $\left(N_{r}\right)$ and the mixed convection parameter $\left(\mathrm{Ra}_{x} / \mathrm{Pe}_{x}\right)$ on the temperature distribution $\theta(\eta)$ at $N_{b}=0.5, N_{t}=0.5$, Le $=10$, and $\alpha=\pi / 6$.

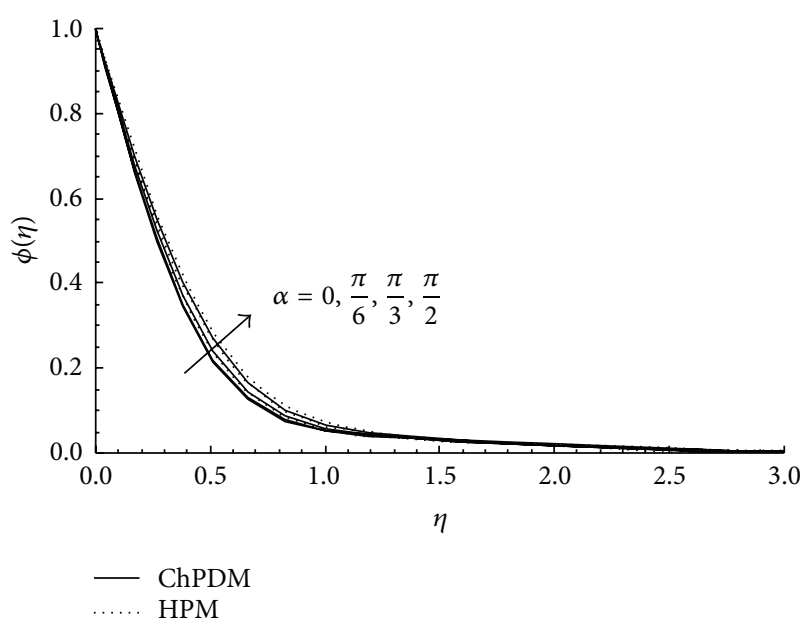

Figure 4: Comparison of ChPDM (solid) and HPM (dotted) for the effect of the angle of inclination $(\alpha)$ on the nanoparticle concentration distribution $\phi(\eta)$ at $N_{b}=0.5, N_{t}=0.5, N_{r}=0.5$, $\mathrm{Le}=10$, and $\mathrm{Ra}_{x} / \mathrm{Pe}_{x}=0.5$.

$\alpha=\pi / 6$, where the slight difference between the curves increases as Lewis number Le decreases.

From the above comparisons, one can conclude that although slight differences are observed between the two approaches in some cases, two terms approximate solutions for $\theta(\eta)$ and $\phi(\eta)$ are still effective and of course can be enhanced via adding more terms for the homotopy series solutions. Accordingly, the HPM can be used as a method of solution with highly trust for investigating several similar models by following the proposed analysis of the current paper.

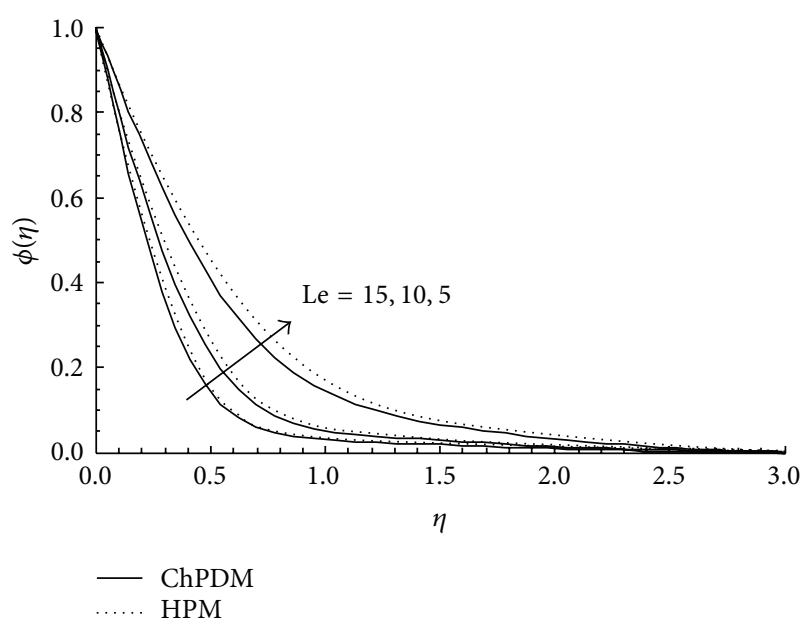

FIGURE 5: Comparison of ChPDM (solid) and HPM (dotted) for the effect of the Lewis number (Le) on the nanoparticle concentration distribution $\phi(\eta)$ at $N_{b}=0.5, N_{t}=0.5, N_{r}=0.5, \mathrm{Ra}_{x} / \mathrm{Pe}_{x}=0.5$, and $\alpha=\pi / 6$.

\section{Conclusion}

In the present paper, the system of differential equations, describing the mixed convection boundary-layer nanofluids flow along an inclined plate embedded in a porous medium, has been successfully solved by applying two methods, analytically by the homotopy perturbation and numerically by Chebyshev pseudospectral differentiation matrix, which was used as a control technique. In order to obtain meaningful physical solution, a new way was deduced to formulate the deformed system of differential equations. Accordingly, the approximate solution was expressed in terms of an entire error function. This proposed way of applying HPM may be of interest for investigating similar models in the future. On applying HPM, it was found that two terms of the homotopy series are enough to achieve very good solutions when compared with the accurate results obtained numerically via ChPDM. This was investigated for various values of the appropriated physical parameters: temperature distribution $\theta(\eta)$ and nanoparticle concentration distribution $\phi(\eta)$ at variation of the thermophoretic parameter $\left(N_{t}\right)$, Brownian motion parameter $\left(N_{b}\right)$, buoyancy ratio parameter $\left(N_{r}\right)$, angle of inclination $(\alpha)$, Lewis number (Le), and mixed convection parameter $\left(\mathrm{Ra}_{x} / \mathrm{Pe}_{x}\right)$.

\section{Acknowledgment}

This project was funded by the Deanship of Scientific Research (DSR), King Abdulaziz University, Jeddah, under grant no. (233/965/1433). The authors, therefore, acknowledge with thanks DSR technical and financial support.

\section{References}

[1] S. U. S. Choi, "Enhancing thermal conductivity of fluids with nanoparticles," in Proceedings of the ASME International 
Mechanical Engineering Congress and Exposition, pp. 99-105, ASME, San Francisco, Calif, USA, 1995.

[2] S. U. S. Choi, Z. G. Zhang, W. Yu, F. E. Lockwood, and E. A. Grulke, "Anomalous thermal conductivity enhancement in nanotube suspensions," Applied Physics Letters, vol. 79, no. 14, pp. 2252-2254, 2001.

[3] H. Masuda, A. Ebata, K. Teramae, and N. Hishinuma, "Alterlation of thermal conductivity and viscosity of liquid by dispersing ultra-fine particles (Dispersion of g- $\mathrm{Al}_{2} \mathrm{O}_{3} \mathrm{SiO}_{2}$, and $\mathrm{TiO}_{2}$ ultra-fine particles)," Netsu Bussei, vol. 7, pp. 227-233, 1993.

[4] S. Lee, S. U.-S. Choi, S. Li, and J. A. Eastman, "Measuring thermal conductivity of fluids containing oxide nanoparticles," Journal of Heat Transfer, vol. 121, no. 2, pp. 280-288, 1999.

[5] A. V. Kuznetsov and D. A. Nield, "Natural convective boundarylayer flow of a nanofluid past a vertical plate," International Journal of Thermal Sciences, vol. 49, no. 2, pp. 243-247, 2010.

[6] W. A. Khan and I. Pop, "Boundary-layer flow of a nanofluid past a stretching sheet," International Journal of Heat and Mass Transfer, vol. 53, no. 11-12, pp. 2477-2483, 2010.

[7] N. Bachok, A. Ishak, and I. Pop, "Boundary-layer flow of nanofluids over a moving surface in a flowing fluid," International Journal of Thermal Sciences, vol. 49, no. 9, pp. 1663-1668, 2010.

[8] E. H. Aly and A. Ebaid, "New exact solutions for boundarylayer flow of a nanofluid past a stretching sheet," Journal of Computational and Theoretical Nanoscience, vol. 10, no. 4, pp. 2591-2594, 2013.

[9] J.-H. He, "Homotopy perturbation technique," Computer Methods in Applied Mechanics and Engineering, vol. 178, no. 3-4, pp. 257-262, 1999.

[10] J.-H. He, "A coupling method of a homotopy technique and a perturbation technique for non-linear problems," International Journal of Non-Linear Mechanics, vol. 35, no. 1, pp. 37-43, 2000.

[11] J.-H. He, "Homotopy perturbation method: a new nonlinear analytical technique," Applied Mathematics and Computation, vol. 135, no. 1, pp. 73-79, 2003.

[12] J.-H. He, "Comparison of homotopy perturbation method and homotopy analysis method," Applied Mathematics and Computation, vol. 156, no. 2, pp. 527-539, 2004.

[13] J.-H. He, "Asymptotology by homotopy perturbation method," Applied Mathematics and Computation, vol. 156, no. 3, pp. 591596, 2004.

[14] J.-H. He, "Homotopy perturbation method for solving boundary value problems," Physics Letters A, vol. 350, no. 1-2, pp. 8788, 2006.

[15] J.-H. He, "Application of homotopy perturbation method to nonlinear wave equations," Chaos, Solitons and Fractals, vol. 26, no. 3, pp. 695-700, 2005.

[16] P. D. Ariel, "The three-dimensional flow past a stretching sheet and the homotopy perturbation method," Computers \& Mathematics with Applications, vol. 54, no. 7-8, pp. 920-925, 2007.

[17] S. Pamuk and N. Pamuk, "He's homotopy perturbation method for continuous population models for single and interacting species," Computers \& Mathematics with Applications, vol. 59, no. 2, pp. 612-621, 2010.

[18] H. Aminikhah, "The combined Laplace transform and new homotopy perturbation methods for stiff systems of ODEs," Applied Mathematical Modelling, vol. 36, no. 8, pp. 3638-3644, 2012.
[19] A. H. Nayfeh, Perturbation Methods, John Wiley \& Sons, New York, NY, USA, 1973.

[20] E. H. Aly, M. Benlahsen, and M. Guedda, "Similarity solutions of a MHD boundary-layer flow past a continuous moving surface," International Journal of Engineering Science, vol. 45, no. 2-8, pp. 486-503, 2007.

[21] M. Guedda, E. H. Aly, and A. Ouahsine, "Analytical and ChPDM analysis of MHD mixed convection over a vertical flat plate embedded in a porous medium filled with water at $4^{\circ} \mathrm{C}$," Applied Mathematical Modelling, vol. 35, no. 10, pp. 5182-5197, 2011.

[22] E. H. Aly and A. Ebaid, "On the exact analytical and numerical solutions of nano boundary-layer fluid flows," Abstract and Applied Analysis, vol. 2012, Article ID 415431, 22 pages, 2012.

[23] R. A. van Gorder, E. Sweet, and K. Vajravelu, "Nano boundary layers over stretching surfaces," Communications in Nonlinear Science and Numerical Simulation, vol. 15, no. 6, pp. 1494-1500, 2010.

[24] P. Rana, R. Bhargava, and O. A. Bég, "Numerical solution for mixed convection boundary layer flow of a nanofluid along an inclined plate embedded in a porous medium," Computers \& Mathematics with Applications, vol. 64, no. 9, pp. 2816-2832, 2012.

[25] M. Abramowitz and I. A. Stegun, Handbook of Mathematical Functions, Dover, New York, NY, USA, 4th edition, 1972.

[26] E. M. E. Elbarbary and S. M. El-Sayed, "Higher order pseudospectral differentiation matrices," Applied Numerical Mathematics, vol. 55, no. 4, pp. 425-438, 2005. 


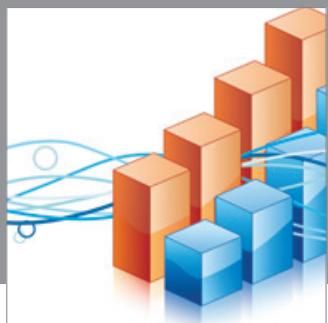

Advances in

Operations Research

mansans

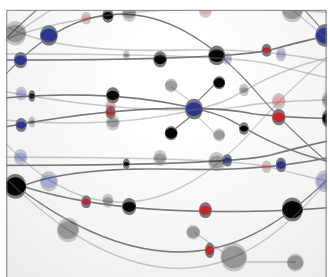

The Scientific World Journal
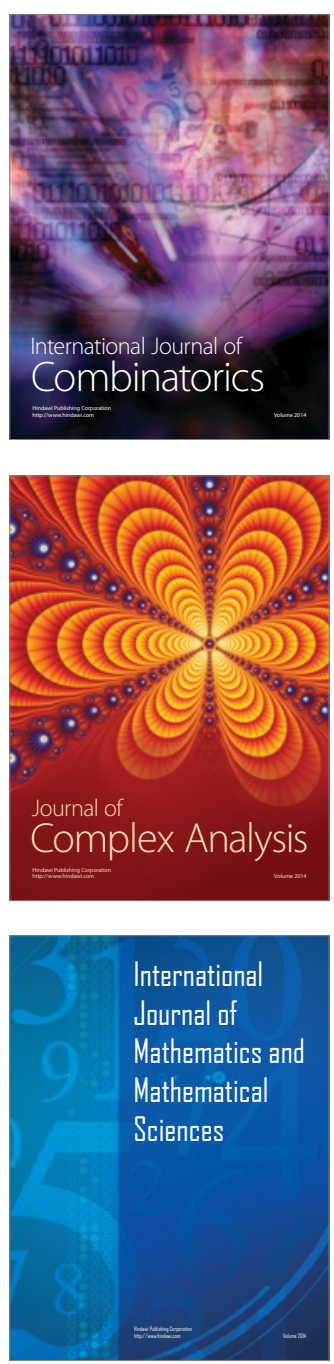
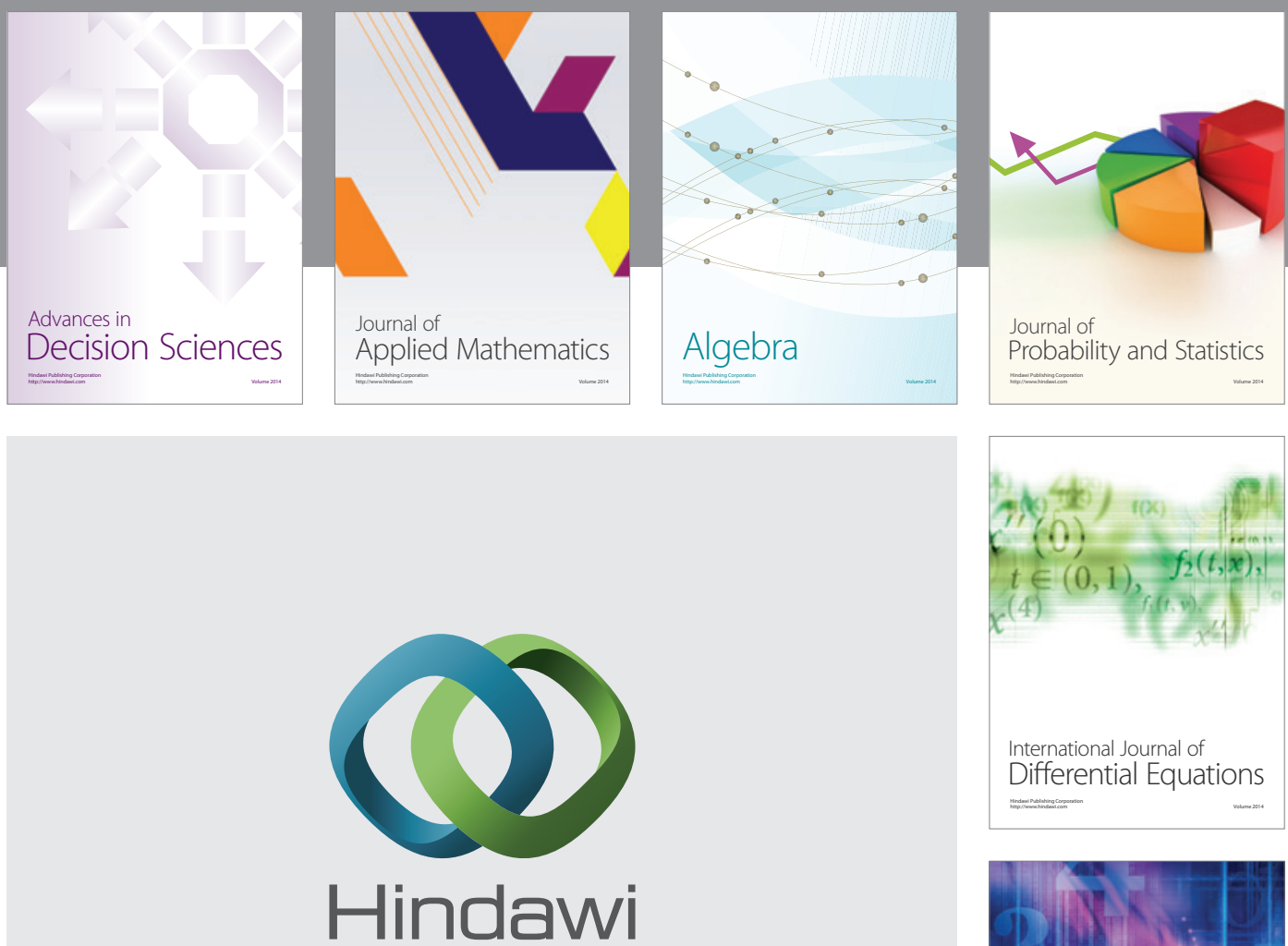

Submit your manuscripts at http://www.hindawi.com
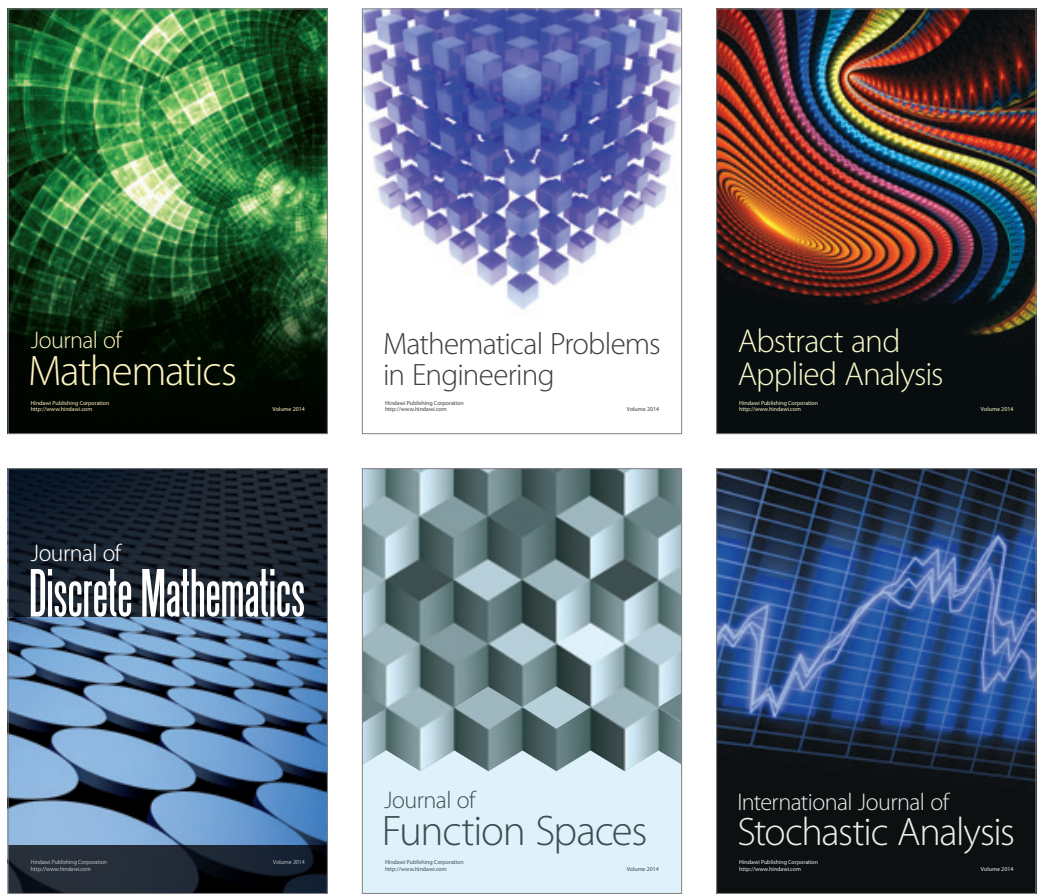

Journal of

Function Spaces

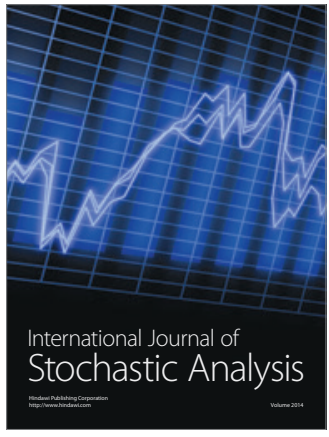

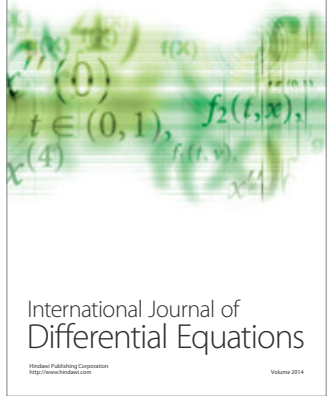
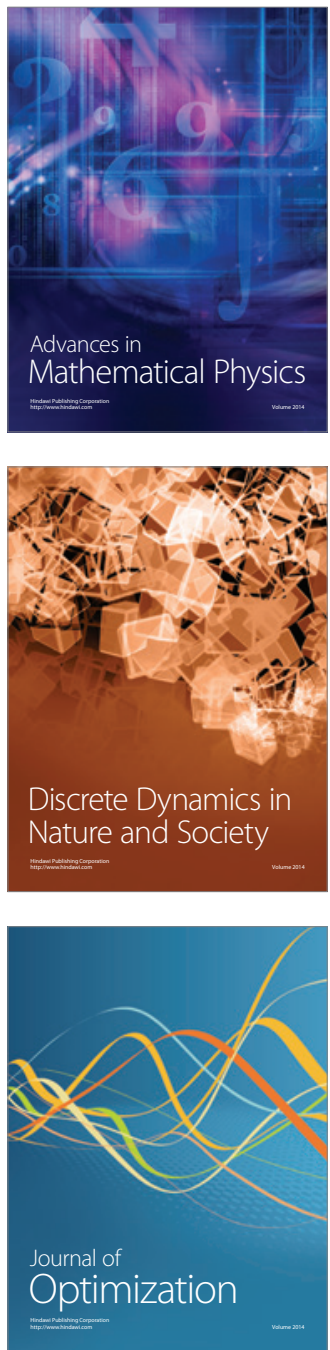